\title{
DESIGN, SIMULATION AND EXPERIMETAL TESTS OF HYDRAULIC HYDRO-SOUNDER
}

\author{
Tapio VIRVALO*, Arto LAINE** \\ *IHA, Tampere University of Technology, Finland \\ P.O.Box 589, 33101 Tampere \\ Tel. -358-3-31152262, Fax. -358-3-31152240 \\ e-mail tapio.virvalo@tut.fi \\ ${ }^{* *}$ Patria Advanced Solutions Oy \\ Naulakatu 3, 33100 Tampere, Finland
}

\begin{abstract}
Mines installed into sea are a serious threat to navigation during wars or other un-certain conditions. During years mines have become quite sophisticated. Every ship passing through the water emits sound, disturbs the Earth's magnetic field, and causes fluctuations in water pressure. Mine clearing is important and challenging task. Sea mines react to stimulus of ships. Noise caused by ships is one of remarkable stimulus. Mines reacting to noise are tried to clear with acoustic mine clearing equipment. In order to image noise generated by different ships quite large frequency range is required. High frequencies $(>100 \mathrm{~Hz})$ can be achieved, for instance, with permanent magnet and magnetostrictive hydro-sounders. At low frequencies $(\leq 60 \mathrm{~Hz})$ hydraulic hydro-sounder might be a reasonable solution. In this paper the preliminary design, modeling and experimental tests of hydraulic cylinder driven hydro-sounder are presented.
\end{abstract}

\section{KEYWORDS}

Hydro-sounder, hydraulic servo

\section{INTRODUCTION}

Mines installed into sea are a serious threat to navigation during wars or other un-certain conditions. During years mines have become quite sophisticated. Yesterday's moored or drifting contact mine is still a threat, but today's modern naval arsenal also includes the bottom or moored influence mine. The influence mine lies on the bottom or floats at the end of its tether and waits...sometimes for weeks or months. Every ship passing through the water emits sound, disturbs the Earth's magnetic field, and causes fluctuations in water pressure. Mines can be built and programmed to detect all of these changes. If an approaching ship emits enough noise, causes a sufficiently strong change in the surrounding magnetic field, or generates a significantly large fluctuation in 
water pressure, it can cause the influence mine to detonate. Mine clearing is important and challenging task. Sea mines react to stimulus of ships. Noise caused by ships is one of remarkable stimulus. Mines reacting to noise are tried to clear with acoustic mine clearing equipment.

In order to image noise generated by different ships quite large frequency range is required. High frequencies $(>$ $100 \mathrm{~Hz}$ ) can be achieved, for instance, with permanent magnet and magnetostrictive hydro-sounders, Fig.1.

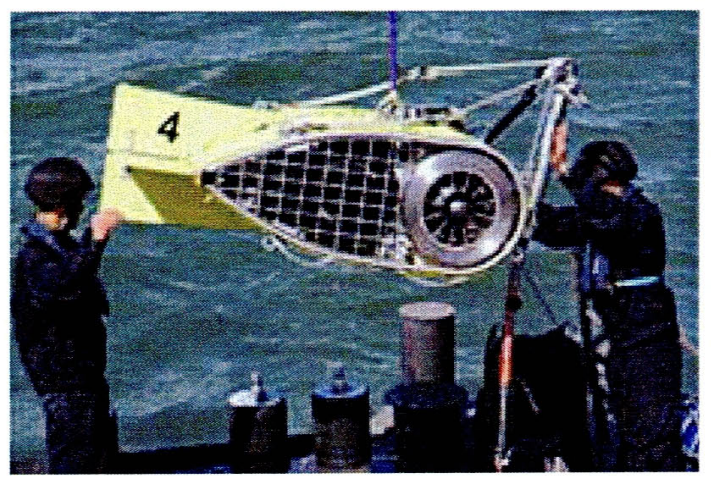

Figure 1. An example of magnetic hydro-sounder At low frequencies $(\leq 60 \mathrm{~Hz})$ hydraulic hydro-sounder might be a reasonable solution. The principle idea of the hydraulic test system is shown in Fig.2.

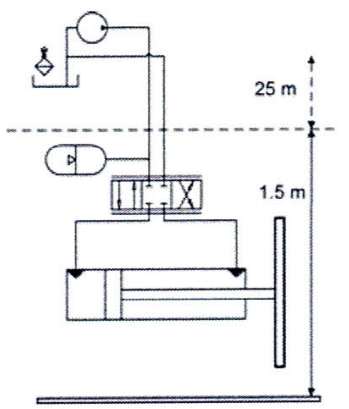

Figure 2, Basic idea of hydraulic hydro-sounder test system

The plate is moved by the hydraulic cylinder under water generating pressure waves. For practical reasons the initial feasibility tests are decided to realize in the laboratory where reasonable size water spool exits. The size of the spool is $6 \times 5 \times 10$ (length, width and high). Based on experiences of preliminary tests with other types of hydro-sounders the diameter of the moving plate is selected $400 \mathrm{~mm}$. Later also the plate of $600 \mathrm{~mm}$ diameter is used.

\section{GOALS OF FIESIBILITY STUDY}

In the preliminary design stage the following specifications are set to $400 \mathrm{~mm}$ plate

* Maximum sin-wave frequency $50 \mathrm{~Hz}$ with the position amplitude $2 \mathrm{~mm}$

* Maximum position amplitude $90 \mathrm{~mm}$ at low frequencies

* Maximum velocity $1 \mathrm{~m} / \mathrm{s}$

* Maximum acceleration $200 \mathrm{~m} / \mathrm{s}^{2}$

Because there is very little information available about the dynamic load of the plate in this kind of application experimental tests are necessary. Before experimental tests some simple modeling and simulations are considered also necessary.

With simulations responses to different amplitudes and frequencies of sin-wave reference signals are studied with some basic controllers. In this way the following issues are tried to find

* Suitable reference signal

$$
\text { * Position }
$$

* Required performance of servo valve

* Size of hydraulic cylinder

* Required supply pressure

* Basic structure of controller

* Specifications of position sensor

After simulations an experimental set-up is realized based on simulations and reasonably available components. The goals of experimental tests are:

* To check performance

* To find forces caused by water

* Inertia load

* Friction forces

* To get information for more detail modeling 


\section{PRELIMINARY MODELLING}

Quite large study of Internet and literature gave very poor results in the modeling of the moving of a round plate in water against its surface. The goal of the modeling is to find suitable abstract model for system and controller design purpose. As the first approximation of modeling interaction of the plate and water is considered as an inertia load and viscous friction. The inertia load is approximated as a mass of cylinder with the diameter of the plate and high of the double of the diameter. This leads to the inertia load of $50 \mathrm{~kg}$. Simple test with the paddle tells that water restricts the movement so far the paddle moves. On the other hand the inertia of water behaves totally different as mechanical inertia. In this stage the behavior of water inertia is modeled as follows:

$$
\begin{aligned}
& \text { if }(\text { vel }>0.0 \& \text { acc }>0.0) \text { then } ! \text { vel=velocity } \\
& \text { wflag }=1.0 ! \text { wflag }=\text { flag } \\
& \text { else if }((\text { vel }>0.0 \& \text { acc }<0.0) \text { then } \text { !acc=acceleration } \\
& \text { wflag }=0.0 \\
& \text { else if }((\text { vel }<0.0 \& \text { acc }<0.0) \text { then } \\
& \text { wflag }=1.0 \\
& \text { else if }((\text { vel }<0.0 \& \text { acc }>0.0) \text { then } \\
& \text { wflag }=0.0 \\
& \text { end if }
\end{aligned}
$$

The effective water inertia is descript as a second order system

$$
\mathrm{m}=\frac{\omega^{2} \cdot \text { wflip }}{\mathrm{s}^{2}+2 \cdot \delta \cdot \omega \cdot \mathrm{s}+\omega^{2}} \cdot \mathrm{m}_{\mathrm{WAT}}
$$

Where $\omega$ is estimated natural frequency of effective water mass (inertia), $\delta$ is natural damping factor of effective water mass (inertia), and $\mathrm{m}_{\mathrm{WAT}}$ is estimated water mass.

Figure 4 shows as an example the responses of water inertia and the acceleration of the plate in an open loop control case. The viscous friction coefficient (force depending on the velocity of the plate) is estimated in this stage relatively high, $7000 \mathrm{Ns} / \mathrm{m}$.

Lot of simulations is done with this water model. According to simulations with this preliminary model the following conclusions can be made:

* It is possible to achieve set performance goals
* Open loop control is nod good enough

* Position drift

* Low bandwidth

* Closed loop velocity control with loose position control outer loop seems promising

* No position drift

* Lower phase sift than closed loop position control

* $\quad$ Feed forward loop improves performance.

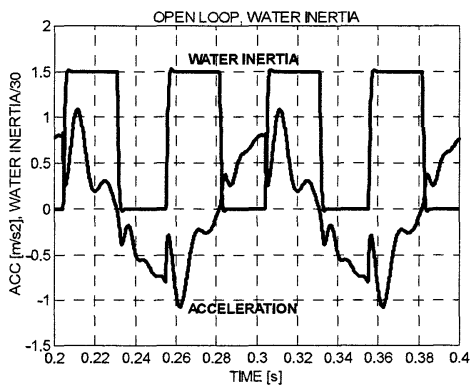

Figure 3. Responses of water inertia and acceleration of plate, simulated

\section{EXPERIMENTAL TESTS}

In order to get more exact information about the behavior and performance of hydraulic hydro-sounders an experimental test set-up realized in the laboratory circumstance.

\section{Specifications of test set-up}

Experimental set-up is realized with already available components, because the purpose of experimental tests is to confirm simulations and get more detail information for improving the water model

* Cylinder 32/18-90 (piston dia., rod dia. stroke [mm])

* Servo valve, nominal flow 401/min with pressure drop 35bar/control notch

* Supply pressure

* Pipes between pump/tank and unit

* Inner diameter $16 \mathrm{~mm}$

* Length $25 \mathrm{~m}$

* Accumulator 8litre

* Analogue position sensor (potentiometer) 
In order to achieve a symmetric structure two hydraulic units are assembled into the same water tight box as shown in Fig.4.

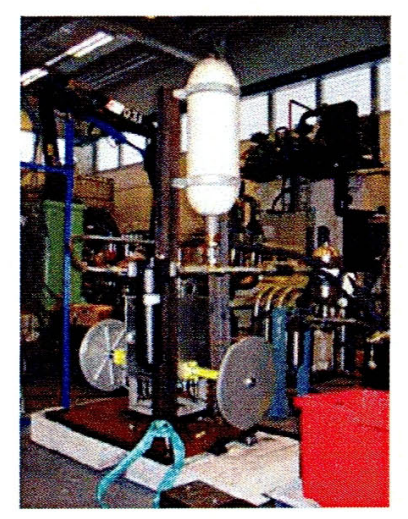

Figure 4. Test unit

\section{Experimental tests}

In order to find the inertia and friction load caused by water open loop measurements are done. Sin-waves with different amplitudes and frequencies are used as reference signals in the performance measurements of the closed loop control system in water. The cylinder chamber pressures, supply and return line pressures as well as the position of the plate are measured in all cases. The test unit is pinned by anchored it into the floor.

\section{Open loop measurements in air}

Firstly open loop measurements are done in air in order to find friction forces of the hydraulic cylinder. The procedure of open loop measurements is

* Fixed start point is achieved with closed loop position control

* Position control is switched off and after short delay the step-wise control signal of the valve is generated

* When the plate reaches the fixed end point the control signal of the valve is switched step-wise off.

According to these measurements the velocity of the plate is a linear function of the valve control signal. The friction force as a function of the plate velocity is presented in Fig.5.

The hydraulic cylinder is normal low-price commercial cylinder and so the friction force is remarkably high, especially at higher velocities.

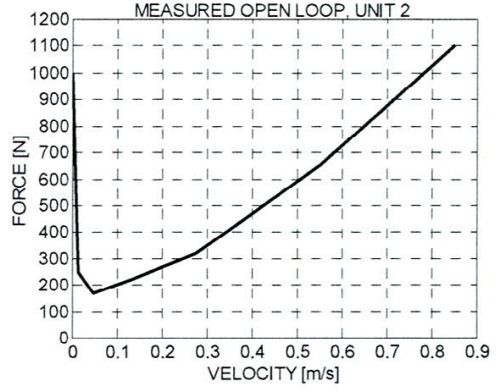

Figure 5. Measured friction force as a function of velocity, in air

\section{Open loop measurements in water}

The same kinds of open loop measurements are done in water. The plate velocity as a function of the valve control signal is shown in Fig.6.

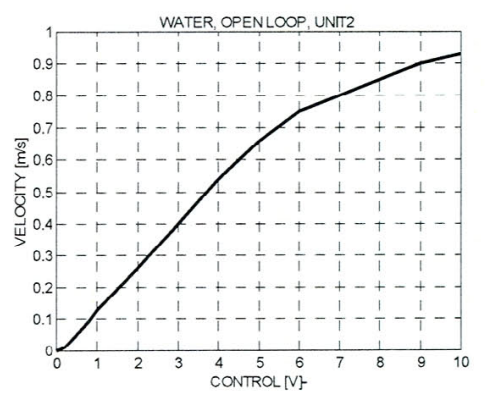

Figure 6. Measured plate velocity as a function of valve control signal (max 10V), in water

The friction force as a function of the plate velocity is presented in Fig.7. The velocity of the plate is not linear function of the control signal any more. Higher velocities than $0.6 \mathrm{~m} / \mathrm{s}$ water causes remarkably higher restrictive force than air. According to these measurements it seems that the velocity depending friction force is a non-linear function of velocity. Some tests done with the higher supply pressure confirm this. According to these preliminary tests it seems that a reasonable approximation is the third order dependence. This estimation is shown in Fig. 8 . 


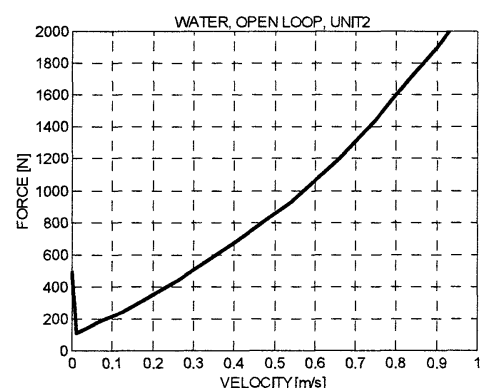

Figure 7. Measured friction force as a function of velocity, in water

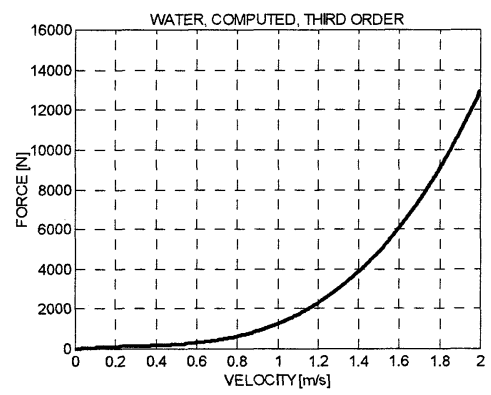

Figure 8. Estimation of friction force as a function of plate velocity in water

Estimation of water inertia is based on the measured cylinder chamber pressures and computed acceleration of the plate. The computed force acting on the plate is shown in Fig.9 and acceleration of the plate in Fig. 10. An interesting phenomenon can be observed in both figures. The force and acceleration/deceleration differ significantly each other.

Based on the same kinds of estimations in air the mechanical part of the inertia can be eliminated and this results water inertia $39 \mathrm{~kg}$. Figure 11 shows, as an example, the response of the return line pressure with maximum step-wise valve control signal. The long return line $(25 \mathrm{~m})$ causes very high pressure peak in the acceleration stage limiting the reachable acceleration in step-wise control. In normal application cases

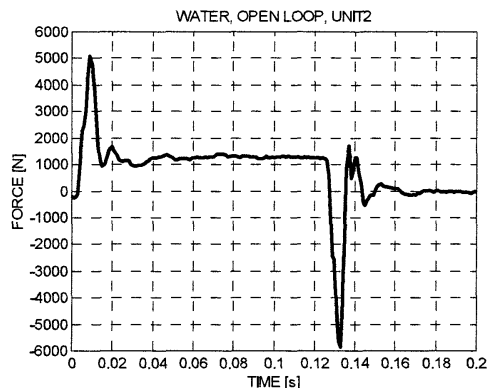

Figure 9. Measured force acting on plate, in water, control $50 \%$

continuously oscillating reference signals are used. As an example, Figure 12 shows the return line pressure when the sin-wave reference signal with the amplitude $0.4 \mathrm{~m} / \mathrm{s}$ and frequency $40 \mathrm{~Hz}$ is used.

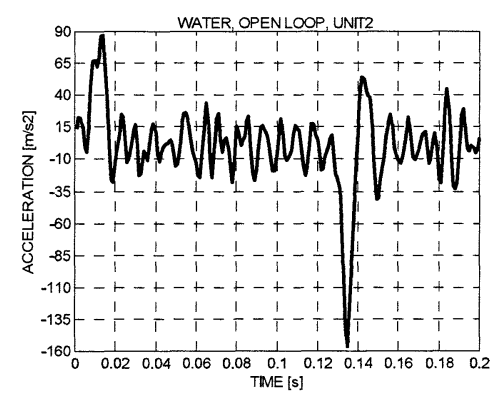

Figure 10. Measured acceleration of plate, in water, control $50 \%$

\section{Closed loop control in water}

The purpose of closed loop control tests is to ensure sufficient performance of hydraulic hydro-sounder. The hydraulic, especially the cylinder and control valve, is not the best one, but a compromise between expected performance and available components.

A linear PI-controller and forward loop is used in these tests. The sampling time is $1 \mathrm{~ms}$ and the tuning is made manually at the frequency of $20 \mathrm{~Hz}$. This tuning is a reasonable compromise, because the best tuning depends on the frequency, especially when forward loop is used. 


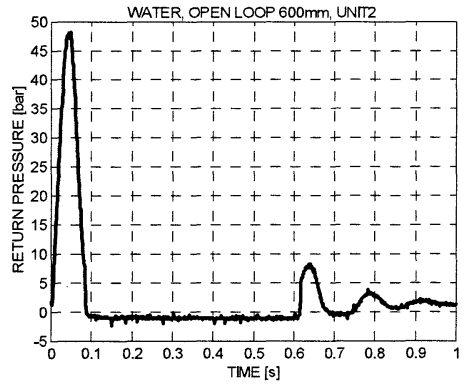

Figure 11. Measured response of return line pressure in step-wise control, control $100 \%$, in water

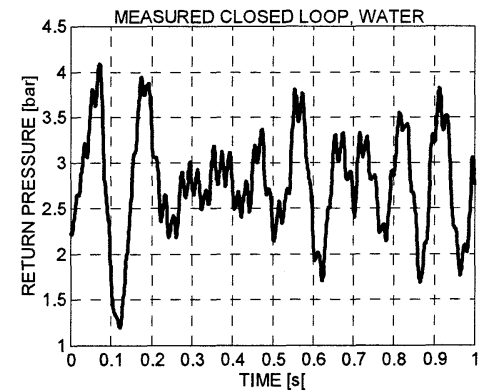

Figure 12. Measured response of return line pressure in sin-wave reference signal, in water

As examples the responses of velocity and position are presented in Fig.13 and 14. The reference signal is a sin-wave with the amplitude $0.4 \mathrm{~m} / \mathrm{s}$ and the frequency $50 \mathrm{~Hz}$. There is the remarkable phase shift $\approx-200^{\circ}$ and also amplitude damping $-3 \mathrm{db}$ in the velocity response, Fig. 13. The performance depends mostly on the dynamic characteristics of the used servo valve. The steady state point of the plate is in the middle of the stroke. The amplitude of the position response is $1 \mathrm{~mm}$ around the steady state point, Fig.14. The return line pressure oscillates with the amplitude about 5 bar.

\section{DISCUSSION AND CONCLUSIONS}

Very rough approximation model of the behavior of water in this kind of application is valid so far that preliminary estimation of performance can be done. Water forms some kind of inertia load, but its behavior is strongly non-linear and does not resemble mechanical inertia load.
Water causes non-linear viscous friction which increases strongly as a function of the plate velocity. Most critical component is the control valve and high response valve is required in order to achieve good performance at frequencies of $50 \ldots 70 \mathrm{~Hz}$. Anyway, achieved results are so promising that $R \& D$ work can be continued.

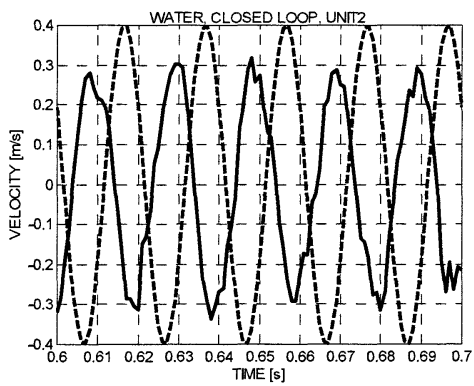

Figure 13. Velocity response (solid line), velocity reference (dash line) $50 \mathrm{~Hz}, 0.4 \mathrm{~m} / \mathrm{s}$ in water

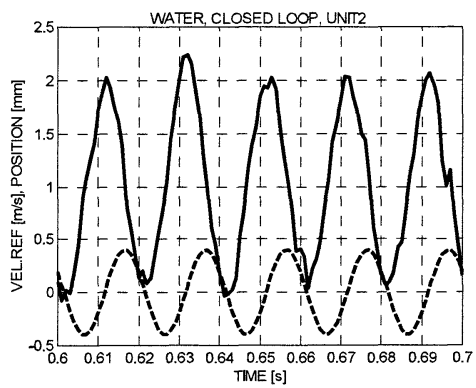

Figure 14. Position response (solid line), velocity reference (dash line) $50 \mathrm{~Hz}, 0.4 \mathrm{~m} / \mathrm{s}$ in water

\section{FURTHER WORKS}

Based on open and closed loop measurements the model of inertia load and friction force of the plate and water combinations could be focused. With this more accurate model the required performance of the hydraulic cylinder and servo valve could be specified. Different control algorithms could also be studied effectively with the high quality model.

Experimental tests in real circumstances are also necessary after simulation and maybe further laboratory tests. 